\title{
Integrating MRI and Chemokine Receptor CXCR4-Targeted PET for Detection of Leukocyte Infiltration in Complicated Urinary Tract Infections After Kidney Transplantation
}

\author{
Thorsten Derlin*1, Faikah Gueler*2, Jan Hinrich Bräsen ${ }^{3}$, Jessica Schmitz ${ }^{3}$, Dagmar Hartung ${ }^{4}$, Thomas R. Herrmann ${ }^{5}$, \\ Tobias L. Ross ${ }^{1}$, Frank Wacker ${ }^{4}$, Hans-Jürgen Wester ${ }^{6}$, Marcus Hiss ${ }^{2}$, Hermann Haller ${ }^{2}$, Frank M. Bengel ${ }^{1}$, \\ and Katja Hueper ${ }^{4}$
}

${ }^{1}$ Department of Nuclear Medicine, Hannover Medical School, Hannover, Germany; ${ }^{2}$ Department of Nephrology, Hannover Medical School, Hannover, Germany; ${ }^{3}$ Institute of Pathology, Hannover Medical School, Hannover, Germany; ${ }^{4}$ Department of Radiology, Hannover Medical School, Hannover, Germany; ${ }^{5}$ Department of Urology and Urological Oncology, Hannover Medical School, Hannover, Germany; and ${ }^{6}$ Radiopharmaceutical Chemistry, Technical University of Munich, Munich, Germany

\begin{abstract}
Complicated urinary tract infections (UTIs) are frequent in immunosuppressed patients after kidney transplantation and may lead to allograft failure or urosepsis. Noninvasive detection of allograft involvement as well as localization of the primary site of infection are challenging. Therefore, we sought to determine whether molecularly targeted PET, combined with diffusion-weighted MRI, enables detection of leukocytes in renal allografts. Methods: Thirteen kidney transplant recipients with complicated UTIs underwent both PET with a specific CXCR4 ligand, ${ }^{8} \mathrm{Ga}$-pentixafor, and diffusion-weighted MRI. The spatial distribution and intensity of CXCR4 upregulation in renal allografts as determined by SUVs on PET and diffusion restriction as determined by apparent diffusion coefficients (ADCs) on MRI were analyzed and compared with urinalysis, clinical chemistry and bacteriology, and biopsy, if available. Results: Combined PET/MRI detected acute allograft infection in 9 patients and lower UTI/nonurologic infections in the remaining 4 patients. Leukocyte infiltration was identified by areas of CXCR4 upregulation compared with unaffected parenchyma in PET (SUV mean $_{\text {, }} 4.6$ vs. 3.7; $P<0.01$ ), corresponding to areas with increased cell density in MRI (ADC ${ }_{\min }, 0.89$ vs. $1.59 \times 10^{-3} \mathrm{~mm}^{2} / \mathrm{s}, P<$ 0.01). Allograft CXCR4 signal was paralleled by CXCR4 upregulation in lymphoid organs. Histopathologic evaluation supported a correlation between CXCR4 signal and presence of leukocytes. Conclusion: Combined CXCR4-targeted PET/MRI with ${ }^{68} \mathrm{Ga}$-pentixafor may enable the noninvasive detection of leukocytes in renal allografts. This novel methodology may refine the characterization of infectious and inflammatory kidney diseases and may serve as a platform for future clinical studies targeting allograft infection.
\end{abstract}

Key Words: positron emission tomography (PET); magnetic resonance imaging (MRI); pentixafor; CXCR4; urinary tract infection (UTI)

J Nucl Med 2017; 58:1831-1837

DOI: 10.2967/jnumed.117.193037

Received Mar. 9, 2017; revision accepted Apr. 11, 2017.

For correspondence or reprints contact: Thorsten Derlin, Department of Nuclear Medicine, Hannover Medical School, Carl-Neuberg-Strasse 1, D-30625 Hannover, Germany.

E-mail: Derlin.Thorsten@mh-hannover.de

${ }^{*}$ Contributed equally to this work.

Published online Apr. 27, 2017.

COPYRIGHT @ 2017 by the Society of Nuclear Medicine and Molecular Imaging.
$\mathbf{U}$ rinary tract infection (UTI) is a common complication after renal transplantation $(1,2)$. Multiple episodes of UTIs may occur despite adequate therapy in up to $27 \%$ of allograft recipients and contribute to chronic loss of graft function (3). In immunosuppressed patients, all UTIs are considered complicated. The clinical presentation of UTI may range from asymptomatic bacteriuria to acute graft pyelonephritis (4), or even urosepsis (3-7). Because of the heterogeneity of clinical presentation under immunosuppression and the severity of UTI, diagnosis and treatment remain challenging. The use of noninvasive imaging to detect allograft involvement and to localize the origin of infection would represent a major clinical advance for diagnosis and treatment of complicated UTIs after kidney transplantation.

Recently, a promising C-X-C chemokine receptor 4 (CXCR4)specific ligand, ${ }^{68} \mathrm{Ga}$-pentixafor, has become available for clinical PET/CT $(8,9)$. CXCR4 is a transmembrane receptor involved in the coordinated trafficking and homing of leukocytes between bone marrow and sites of infection (10). ${ }^{68} \mathrm{Ga}$-pentixafor has previously been applied to visualize CXCR-positive $\left(\mathrm{CXCR}^{+}\right)$bone marrowderived cells in myeloproliferative diseases $(11,12) .{ }^{68} \mathrm{Ga}$-pentixafor has also been reported to specifically identify postinfarction myocardial inflammation mediated by CXCR4 ${ }^{+}$leukocytes (13) and atherosclerotic plaque inflammation (14). Diffusion-weighted MRI has been shown to provide useful information for diagnosing and monitoring pyelonephritis foci in transplanted kidneys (15). MRI-derived apparent diffusion coefficients (ADCs) are inversely correlated with inflammatory cell infiltration in the kidneys (16). However, ADCs are also reduced in areas of graft fibrosis and cannot distinguish between active infectious foci and scarring $(16,17)$.

Therefore, we hypothesized that CXCR4-targeted PET/CT, combined with diffusion-weighted MRI, enables robust and specific detection of leukocytes in renal allografts and facilitates the differential diagnosis of infection. This hypothesis was tested in patients with complicated UTIs after kidney transplantation.

\section{MATERIALS AND METHODS}

Patients and Clinical Parameters

Thirteen consecutive patients ( 6 men, 7 women; median age, 56.9 y; interquartile range [IQR], 43.3-60.9 y) with a history of renal 
transplantation and complicated UTIs underwent both ${ }^{68} \mathrm{Ga}$-pentixafor PET/CT and diffusion-weighted MRI within a median of $0.5 \mathrm{~d}$ (IQR, 0-2 d), when admitted due to clinically suspected recurrent UTI. Noninvasive imaging was performed for clinical purposes, that is, to determine allograft involvement and to identify the origin of infection. ${ }^{68} \mathrm{Ga}$-pentixafor was used clinically according to $\S 13.2 \mathrm{~b}$ of the German Pharmaceuticals Act. The institutional review board approved this retrospective study, and the requirement to obtain informed consent was waived.

For validation of imaging procedures, a panel of laboratory parameters including serum creatinine, estimated glomerular filtration rate (eGFR) according to the Chronic Kidney Disease Epidemiology Collaboration formula (18), and systemic inflammatory parameters (C-reactive protein levels [CRP] and blood leukocyte count) were determined at admission, before and after treatment. The diagnosis of UTI was established on the basis of urinalysis and microbiologic evaluation of spot urine samples. Allograft biopsy was performed in 2 patients when additional rejection was suspected due to acute transplant failure. The final clinical diagnosis was established by a senior attending nephrologist and recorded for the analysis.

\section{PET/CT Acquisition and Image Reconstruction}

${ }^{68} \mathrm{Ga}$-pentixafor (CPCR4.2) was synthesized as previously described $(19,20)$ using a ${ }^{68} \mathrm{Ge} /{ }^{68} \mathrm{Ga}$ generator (Eckert \& Ziegler) connected to an automated module (Scintomics). All studies were conducted using a dedicated PET/CT system (Biograph mCT 128 Flow; Siemens), equipped with an extended field-of-view lutetium oxyorthosilicate PET component and a 128-slice spiral CT component. The patients received an intravenous injection of $136 \mathrm{MBq}$ (IQR, 122-148) of ${ }^{68} \mathrm{Ga}$-pentixafor. At $15 \mathrm{~min}$ after injection, each patient received $0.5 \mathrm{mg}$ of furosemide per kilogram of body weight (maximum, $40 \mathrm{mg}$ ) followed by oral hydration with $1.5 \mathrm{~L}$ of water. Both procedures were performed to favor tracer excretion and to reduce urinary activity in the renal pelvis, facilitating the analysis of renal parenchyma. Images were acquired directly after the last voiding of the bladder. At 60 min after injection, imaging began with a low-dose non-enhanced helical CT $(120 \mathrm{kV}$; mA modulated; pitch, 1.2; reconstructed axial slice thickness, $5.0 \mathrm{~mm}$ ) performed for attenuation correction. Whole-body PET images were then acquired using continuous bed motion (at a speed of $0.9 \mathrm{~mm} / \mathrm{s}$ for chest and abdomen and $2.1 \mathrm{~mm} / \mathrm{s}$ for legs). All studies were reconstructed using time-of-flight and point-spread function information combined with an iterative algorithm (Ultra HD; Siemens Healthcare; 2 iterations, 21 subsets; matrix, $200 \times 200$; zoom, 1.0; gaussian filter, 5.0).

\section{MRI Acquisition}

MRI was performed on a 1.5-T scanner (Magnetom Aera; Siemens Healthcare) using a combination of two 18-channel phased array bodycoils placed on the upper abdomen and the pelvis and the spine coil. Respiratory-triggered T2-weighted turbo spin echo and breathhold T1weighted vibe sequences were acquired in transverse and oblique coronal planes and covered the entire abdomen. In addition, a respiratorytriggered fat-saturated echoplanar diffusion-weighted imaging sequence was obtained in coronal orientation with the following sequence parameters: field of view, $400-440 \times 400-440 \mathrm{~mm}$; matrix, $128 \times 130$; interpolated matrix, $256 \times 260$; slice thickness, $5 \mathrm{~mm}$; repetition time, 4,000 ms; echo time, $68 \mathrm{~ms}$; 10 b-values, 0, 50, 100, 200, 300, 400, $500,600,800,1,000 \mathrm{~s} / \mathrm{mm}^{2}$; acquisition time, approximately $8 \mathrm{~min}$.

\section{Image Analysis}

PET and MR images were analyzed by a senior nuclear medicine physician (10 y of experience in PET imaging) and a senior radiologist ( $8 \mathrm{y}$ of experience in genitourinary MRI) masked to the results of laboratory tests and microbiologic evaluation in consensus. To that end, a side-by-side comparison of PET and MR images was performed first. Afterward, PET images and ADC maps were fused manually, and the presence and localization of infectious foci were determined and compared.

PET/CT. Transaxial ${ }^{68} \mathrm{Ga}$-pentixafor PET, CT, and fused PET/CT images were analyzed on a dedicated workstation (syngo.via; Siemens Healthcare). For identification of upregulated CXCR4 expression in renal parenchyma, PET images were visually evaluated for the presence of increased radiotracer uptake $\left(\mathrm{CXCR} 4^{+}\right)$. The localization of these areas was facilitated by concomitant use of PET/CT fusion images. Signal intensities in target regions and renal parenchyma were quantified using $\mathrm{SUV}_{\text {mean }}$ and $\mathrm{SUV}_{\text {max }}$ obtained by manually placing an individual circular volume of interest around lesions and into parenchyma, respectively. Additionally, mean SUVs were also obtained for lumbar vertebra bone marrow and spleen using volumes of interest of 2-cm diameter, as a measure of the systemic inflammatory response, and for regional lymph nodes.

MRI. MR images were analyzed using commercially available Visage software (version 7.1.10; Visage Imaging $\mathrm{GmbH}$ ) and open source Horos software (version 2.0.1; https://www.horosproject.org/). Morphologic abnormalities were documented by a radiologist. Parameter maps of ADC were calculated inline using a monoexponential fit. On parameter maps, the number of foci with visible $\mathrm{ADC}$ reduction was recorded. Minimum $\left(\mathrm{ADC}_{\min }\right)$ and mean $\mathrm{ADC}\left(\mathrm{ADC}_{\text {mean }}\right)$ of target lesions with $\mathrm{ADC}$ reduction and kidney transplant parenchyma were quantified in manually selected regions of interest.

\section{Histopathology}

Kidney biopsy at the time of PET was available in 2 patients and was analyzed by an experienced nephropathologist. Formaldehyde-fixed paraffin-embedded serial sections $(3 \mu \mathrm{m})$ were stained with hematoxylin and eosin, periodic acid-Schiff, Jones' methenamine silver, and anti$\mathrm{C} 4 \mathrm{~d}$ and anti-SV40 for routine diagnostics. Additional immunostaining was conducted with an automated platform (Ventana ULTRA; Ventana Medical Systems) using the following antibodies: polyclonal rabbit anti-CD3 to detect T-lymphocytes, mouse anti-CD20cy (clone L26 for B-lymphocytes), and anti-CD68 (clone PG-M1, for macrophages; all from Dako) and CD15 (clone MMA for granulocytes; BD Biosciences).

\section{Effect of Integrated CXCR4-Targeted Imaging on Patient Management}

Clinicians were asked to specify the preimaging intended treatment. Preimaging intended management and postimaging treatment were compared.

\section{Statistical Analysis}

Categoric variables are presented with absolute and relative frequencies. Continuous variables are expressed as median with interquartile range (IQR). Imaging findings (ADC, SUV) in renal foci in acute allograft pyelonephritis were compared with unaffected parenchyma using Wilcoxon matched-pairs signed-rank tests. Laboratory parameters of patients with and without allograft pyelonephritis were compared using the unpaired Mann-Whitney test. Imaging findings (ADC, SUV) were correlated with clinical parameters using Spearman correlation. Stepwise multiple-regression analysis was performed to identify independent variables for renal CXCR4 signal. Statistical significance was established for $P$ values of less than 0.05 . Data were analyzed using Microsoft Excel 2010 (Microsoft Corp.). Statistical analysis was performed using GraphPad Prism (version 6; GraphPad Software) or MedCalc (version 17.2; MedCalc Software).

\section{RESULTS}

\section{Patient Characteristics and Clinical Parameters}

Relevant patient characteristics are shown in Table 1. Three patients had retransplantation with the nonfunctioning transplant 
TABLE 1

Characteristics of Study Population

\begin{tabular}{|c|c|}
\hline Parameter & Value \\
\hline No. of patients & 13 \\
\hline \multicolumn{2}{|l|}{ Sex } \\
\hline Male & 6 \\
\hline Female & 7 \\
\hline Age (y) & 56.9 (range, 43.3-60.9) \\
\hline \multicolumn{2}{|l|}{ Cause of kidney transplantation } \\
\hline Autosomal dominant polycystic kidney disease & $4 / 13$ \\
\hline Glomerulonephritis & $2 / 13$ \\
\hline Cystic kidney degeneration & $1 / 13$ \\
\hline Diabetic nephropathy & $1 / 13$ \\
\hline IgA nephropathy & $1 / 13$ \\
\hline Acute kidney injury & $1 / 13$ \\
\hline Congenital kidney hypoplasia & $1 / 13$ \\
\hline Nephrosclerosis & $1 / 13$ \\
\hline Refluxnephropathy with recurrent UTI & $1 / 13$ \\
\hline \multicolumn{2}{|l|}{ Transplantation details } \\
\hline Living donor transplantation & $7 / 13$ \\
\hline Second transplantation & $3 / 13$ \\
\hline Time after transplantation $(\mathrm{y})$ & $6.2(3.3-9.5)$ \\
\hline \multicolumn{2}{|l|}{ Laboratory values during infection } \\
\hline Serum leukocytes $\left(10^{3} / \mu \mathrm{L}\right)$ & $8.4(5.9-11.6)$ \\
\hline Serum CRP (mg/L) & $48.6(5.2-116.9)$ \\
\hline Serum creatinine $(\mu \mathrm{mol} / \mathrm{L})$ & $253(187-359)$ \\
\hline eGFR (mL/min/1.73m²) & $22.0(14.5-28.0)$ \\
\hline Urine leukocytes (cells/ $\mu \mathrm{L}$ ) & $29(8-864)$ \\
\hline \multicolumn{2}{|l|}{ Laboratory values after therapy } \\
\hline Serum leukocytes $\left(10^{3} / \mu \mathrm{L}\right)$ & $8.7(4.7-10.9)$ \\
\hline Serum CRP (mg/L) & $5.7(1.6-39.5)$ \\
\hline Serum creatinine $(\mu \mathrm{mol} / \mathrm{L})$ & $204(160-335)$ \\
\hline eGFR (mL/min/1.73m²) & $23.0(15.0-35.0)$ \\
\hline Urine leukocytes (cells/ $\mu \mathrm{L}$ ) & $9(1-540)$ \\
\hline
\end{tabular}

still in situ. Three patients had diabetes mellitus. The median time interval after transplantation was 6.2 y (IQR, 3.3-9.5 y). Eleven patients presented with leukocyturia at the time of imaging. Four patients presented with elevated serum leukocyte count, and 10 patients had elevated serum levels of CRP. Final clinical diagnosis was acute allograft infection in 9 patients and lower UTI in the remaining 4 patients. Microbiologic evaluation revealed single or combined infection with Escherichia coli, Enterococcus faecalis, Pseudomonas aeruginosa, and Candida albicans. In 3 patients under ongoing antibiotic treatment at the time of the imaging, no bacterial growth was detected.

\section{Combined CXCR4-Targeted PET and MRI Specifically Detect CXCR4 ${ }^{+}$Leukocytes in Renal Infection}

In 9 of $13(69 \%)$ patients, patterns consistent with acute allograft infection were concordantly detected on PET and MR images. In these patients, 5 or more focal lesions with significantly reduced minimum and mean $\mathrm{ADC}$ compared with unaffected parenchyma were detected $\left(\mathrm{ADC}_{\min }, 0.89\right.$ [IQR, 0.75-0.96] vs. 1.59 [IQR, 1.56-1.66] $\times 10^{-3} \mathrm{~mm}^{2} / \mathrm{s}, P<0.01 ; \mathrm{ADC}_{\text {mean }}, 1.16$ [IQR, 1.01-1.21] vs. 1.59 [IQR, $1.56-1.66] \times 10^{-3} \mathrm{~mm}^{2} / \mathrm{s}, P<0.01$ ), indicating increased cell density in these areas. These areas of $\mathrm{ADC}$ reduction corresponded to upregulated CXCR4 expression compared with unaffected parenchyma $\left(\mathrm{SUV}_{\max }, 5.9\right.$ [IQR, 3.7-11.5] vs. $\mathrm{SUV}_{\text {mean }}, 3.7$ [IQR, 2.1-4.9], $P<0.01 ; \mathrm{SUV}_{\text {mean }}$, 4.6 [IQR, 2.97.9] vs. 3.7 [IQR, 2.1-4.9], $P<0.01$, Fig. 1), indicating that infiltrating cells detected on MRI were $\mathrm{CXCR} 4^{+}$. One patient showed additional $\mathrm{CXCR} 4^{+}$pulmonary infiltrates indicating pneumonia.

In the other 4 of $13(31 \%)$ patients, PET and MR imaging detected no signs of allograft pyelonephritis, consistent with lower UTI (Fig. 2). Among these patients, whole-body PET imaging 


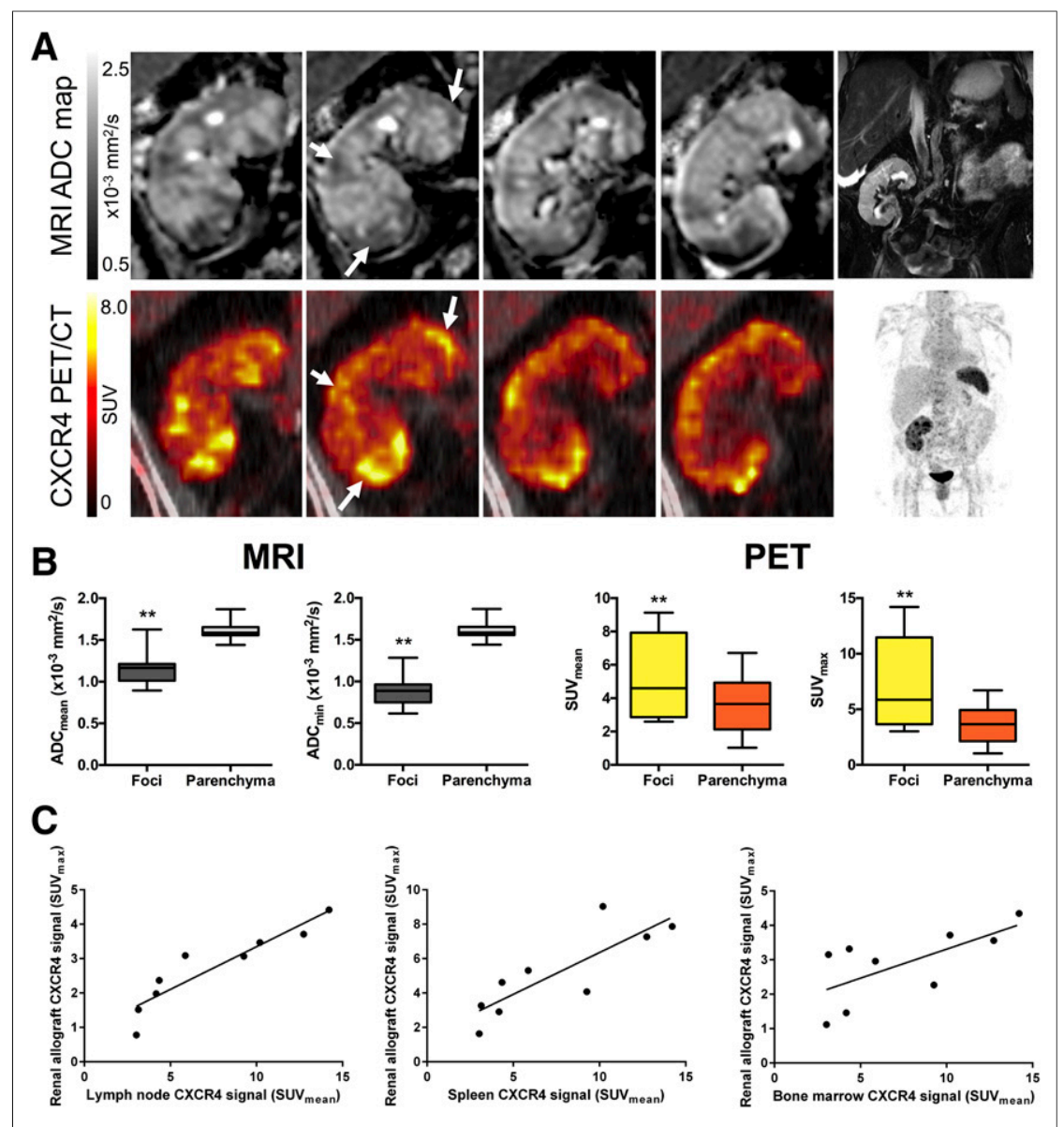

FIGURE 1. PET and MR imaging of acute renal allograft infection. (A) Representative MR and PET images of patient with acute renal allograft infection. ADC maps show areas of reduced ADC (top, frontal views from posterior to anterior) corresponding to foci with upregulated CXCR4 expression at ${ }^{68} \mathrm{Ga}$-pentixafor PET imaging (bottom, arrows). T2-weighted MRI and maximumintensity-projection PET are shown for anatomic orientation. Renal transplant is located in right lower abdomen. Spleen displays high CXCR4 expression due to high content of leukocytes. (B) In addition, box plots of ADC values on MRI and SUV on ${ }^{68} \mathrm{Ga}$-pentixafor PET in CXCR4 ${ }^{+}$foci and unaffected renal transplant parenchyma in 9 patients with acute renal transplant infection are shown. Accumulation of CXCR4 ${ }^{+}$leukocytes was paralleled by CXCR4 upregulation in bone marrow and lymphoid organs (C), highlighting systemic inflammatory response. Bottom and top of box indicate IQR, and band inside box indicates median. Ends of whiskers represent range. ${ }^{* \star} P<0.01$.

revealed additional sites of infection in 3 patients, that is, enhanced CXCR4 expression along the intrahepatic bile ducts indicating acute cholangitis in one patient and within pulmonary infiltrates indicating pneumonia in another patient. One patient presented with acute infection of 2 cysts within the right polycystic kidney (Fig. 3).

\section{CXCR4 $^{+}$Infiltrating Leukocytes Are Associated with Systemic Inflammatory Response}

Detailed data about CXCR4 expression in renal allografts and other organs are shown in Table 2.

In correlation analysis, the signal intensity $\left(\mathrm{SUV}_{\max }\right)$ of $\mathrm{CXCR} 4$ expression in $\mathrm{CXCR}^{+}{ }^{+}$renal parenchymal lesions demonstrated a significant correlation with CXCR4 expression in adjacent lymph nodes $\left(r_{s}=0.92, P<0.001\right)$, spleen $\left(r_{s}=0.83, P=0.005\right)$, and bone marrow $\left(r_{s}=0.67, P=0.04\right)$, highlighting systemic interactions. CXCR4 expression $\left(\mathrm{SUV}_{\max }\right)$ in parenchymal lesions did not exhibit a significant correlation with the level of leukocytes in urine $\left(r_{s}=0.58, P=0.10\right)$ or serum levels of leukocytes $\left(r_{s}=0.33, P=0.38\right)$ and CRP $\left(r_{s}=0.32, P=0.40\right)$. Neither creatinine levels $(P=0.43)$ nor eGFR $(P=$ $0.96)$ were significantly higher in patients with allograft pyelonephritis than in those with UTIs combined with other infections.

In multiple-regression analysis, CXCR4 expression in $\mathrm{CXCR}^{+}{ }^{+}$renal parenchymal lesions was independently associated with CXCR4 expression in regional lymph nodes $(P<0.001)$, but not with serum creatinine levels or eGFR.

\section{Ex Vivo Analysis Confirms Infiltration of Leukocytes in Allograft Infection}

Renal allograft biopsy revealed severe granulomatous tubulointerstitial nephritis with signs of acute kidney injury in 1 patient, and CXCR4-targeted PET and MRI concordantly showed allograft inflammation (Fig. 4). In the other patient, histology did not show signs of acute infection or rejection, and CXCR4-targeted PET and MRI of the allograft were concordantly unremarkable.

\section{Effect of Integrated CXCR4-Targeted Imaging on Patient Management}

Preimaging intended treatment consisted of antibiotic therapy based on antibiotic sensitivity testing. Potential nephrectomy of the native kidneys or the nonfunctioning renal allograft after the first transplantation was discussed when recurrent infections were present and a possible infectious focus in the native kidney or the nonfunctioning allograft was suspected.

In $12(92 \%)$ patients, absence of infectious involvement did not support nephrectomy of the native kidneys or the nonfunctioning allograft. In $1(8 \%)$ patient with infection of the polycystic kidney, the source of infection could be attributed to 1 kidney, and native kidney removal was suggested, but refused by the patient, leading to repetitive antibiotic therapies. In cases with proven transplant pyelonephritis, antibiotic therapy was intensified and treatment was prolonged. In $3(23 \%)$ patients, additional extrarenal sites of infection were detected, influencing the chosen antibiotic regimen.

\section{DISCUSSION}

To our knowledge, this study is the first evaluation of CXCR4targeted PET for visualization of leukocytes in kidney allografts in the context of complicated UTIs. It establishes ${ }^{68} \mathrm{Ga}$-pentixafor as a molecular imaging marker of CXCR4-expressing leukocytes present in renal tissue. Clinical PET/CT, combined with diffusionweighted MRI, may identify upregulated CXCR4 signal of leukocytes in graft pyelonephritis. Histopathologic evaluation supported a correlation between CXCR4 signal and presence of leukocytes.

The CXCR4/CXCL12 axis has a pivotal role in trafficking of leukocytes between bone marrow and sites of infection (10), 


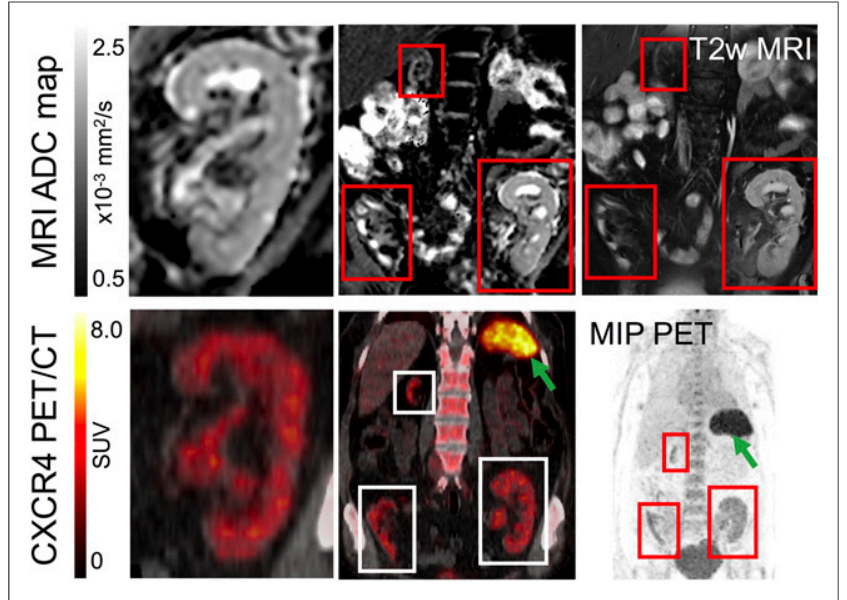

FIGURE 2. PET and MR imaging of patient without renal infection. MR (top) and PET/CT images (bottom) of patient with retransplantation and nephrectomy of left native kidney. Functional renal transplant in left lower abdomen, nonfunctional renal graft with cortical atrophy in right lower abdomen. PET and MRI revealed no signs of infection in all 3 kidneys. Note homogeneously low CXCR4 expression compared with spleen (green arrow) in PET and corresponding homogeneously high $A D C$ values in MRI, excluding acute renal infection. MIP = maximumintensity projection; T2w $=$ T2-weighted.

providing the host with a mechanism to rapidly increase neutrophil delivery to sites of infection via chemokine secretion (21). In previous studies, ${ }^{68} \mathrm{Ga}$-pentixafor has been used to quantify noninfectious inflammation $(13,14)$, linking CXCR4 upregulation to $\mathrm{CD}^{+} 8^{+}$macrophages and $\mathrm{Ly} 6 \mathrm{G}^{+}$granulocytes (13). CXCR4 is expressed by a variety of immune cells including macrophages and neutrophils $(10,21,22)$. In this study, ${ }^{68} \mathrm{Ga}$-pentixafor PET

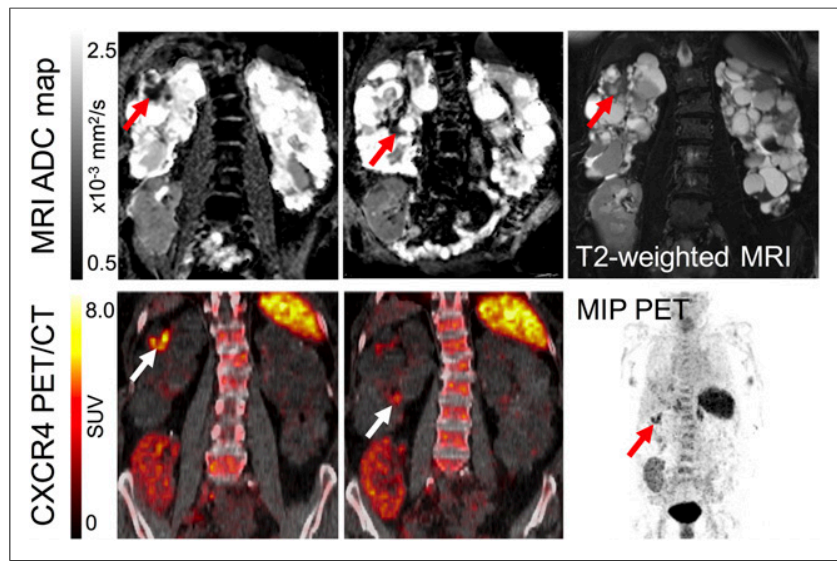

FIGURE 3. PET and MR imaging of acute renal cyst infection. MR (top) and PET/CT images (bottom) of patient with kidney transplantation due to autosomal-dominant polycystic kidney disease and suspected complicated UTI. Imaging revealed upregulated CXCR4 expression in 2 cysts of right native polycystic kidney (white arrow), indicating renal cyst infection. Corresponding MRI confirms a cyst with thick wall (T2-weighted MRI, red arrow) and strong ADC reduction in both areas (red arrows), consistent with PET findings. Allograft in right lower abdomen shows no signs of infection with low homogeneous ${ }^{68} \mathrm{Ga}$-pentixafor signal. MRI shows linear ADC reduction and volume loss, consistent with scarring at upper pole. MIP = maximumintensity projection.
TABLE 2

CXCR4 Expression in Renal Allografts and Other Organs $(n=13)$

\begin{tabular}{|c|c|}
\hline Parameter & Value \\
\hline \multicolumn{2}{|l|}{ Allografts } \\
\hline \multicolumn{2}{|l|}{ CXCR4+ lesions* } \\
\hline $\mathrm{SUV}_{\max }$ & $5.9(3.7-11.5)$ \\
\hline $\mathrm{SUV}_{\text {mean }}$ & $4.6(2.9-7.9)$ \\
\hline \multicolumn{2}{|c|}{ Unaffected parenchyma } \\
\hline$S U V_{\text {mean }}$ & $3.7(2.1-4.9)$ \\
\hline \multicolumn{2}{|l|}{ Other organs } \\
\hline \multicolumn{2}{|l|}{ Lymph nodes } \\
\hline$S U V_{\text {mean }}$ & $3.1(1.8-3.7)$ \\
\hline \multicolumn{2}{|l|}{ Spleen } \\
\hline$S U V_{\text {mean }}$ & $5.0(3.4-7.2)$ \\
\hline \multicolumn{2}{|l|}{ Bone marrow } \\
\hline$S U V_{\text {mean }}$ & $3.2(2.3-3.6)$ \\
\hline
\end{tabular}

${ }^{*} n=9$ patients with focal lesions due to allograft infection.

Values are median with IQR in parentheses.

correctly visualized allograft pyelonephritis in 9 of 13 patients, and detected other previously unknown sites of infection in 3 of 4 other patients with lower UTI, altering the therapeutic management. Whole-body PET/CT was particularly helpful to identify these subclinical infections with atypical presentation under immunosuppression. Allograft biopsy performed in a patient with marked CXCR4 upregulation as determined by PET and cell infiltration as determined by MRI demonstrated corresponding infiltration of leukocytes including granulocytes, macrophages, B cells, and $\mathrm{T}$ cells, underlining that the measured CXCR4 signal represents an inflammatory composite signal, as was to be expected. By contrast, allograft biopsy of a patient without upregulated CXCR4 signal confirmed the absence of inflammatory cell infiltration of renal tissue. This method may hold great potential to provide direct

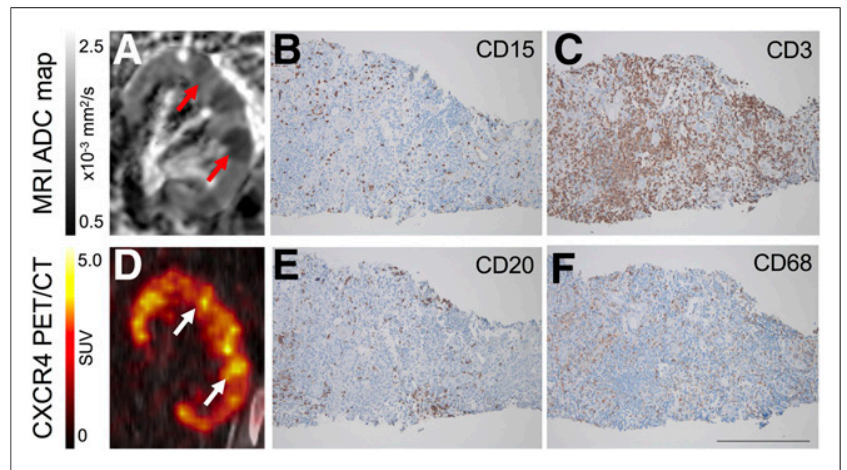

FIGURE 4. Renal leukocyte infiltration in PET and MR imaging. (A) MR image showing focal restriction of diffusion (red arrows). ( $B$ and $C$ ) Biopsy from kidney allograft shows inflammatory cell infiltration typical for allograft infection (granulocytes [CD15, B] and T cells [CD3, C]). (D) Corresponding upregulated CXCR4 expression (white arrows). (E and F) Additional markers of cell infiltration (B cells [CD20, E] and macrophages [CD68, F]). Scale bar indicates $200 \mu \mathrm{m}$. 
insights into the extent, distribution, and time course of inflammatory cell infiltrates in renal tissue.

Other nuclear medicine techniques used for imaging of inflammation including radiolabeled autologous leukocytes and antigranulocyte antibodies for scintigraphic imaging or PET with ${ }^{18} \mathrm{~F}$-FDG have not been evaluated systematically for imaging of allograft infections (23). Limited data have suggested cortical ${ }^{18} \mathrm{~F}$ FDG uptake in pyelonephritis (24). Scintigraphic imaging techniques have lower spatial resolution than PET, and partial-volume effects limit the accuracy of these approaches, particularly for assessment of small cortical lesions. In addition, marked renal elimination (e.g., in the case of antibodies) or unfavorable kinetics (in the case of labeled leukocytes) have limited the clinical use of these methods for imaging of renal infections. ${ }^{18} \mathrm{~F}-\mathrm{FDG}$ is excreted via the kidneys and represents a rather unspecific tracer, which accumulates both in immune cells and in normal renal parenchyma. By contrast, ${ }^{68} \mathrm{Ga}$-pentixafor enables a specific visualization of $\mathrm{CXCR}^{+}$leukocytes in the context of renal allograft infection. However, there is also renal excretion of ${ }^{68} \mathrm{Ga}$-pentixafor, causing some background signal in the kidneys; the average tracer uptake $\left(\mathrm{SUV}_{\text {mean }}\right)$ in unaffected parenchyma was 3.7 (IQR, 2.14.9), albeit significantly lower than in $\mathrm{CXCR}^{+}{ }^{+}$lesions. We applied a diuretic protocol to reduce background activities both in the kidneys and in the urine. Previous studies have demonstrated that diuretic imaging significantly reduces urine activity (25), which also facilitated assessment of the kidneys in this study. Furthermore, we demonstrated that the CXCR4 signal was independent of allograft function. In multiple-regression analysis, CXCR4 expression in renal lesions did not demonstrate a significant association with creatinine levels or eGFR. By contrast, CXCR4 expression in allograft infection was paralleled by concomitant CXCR4 upregulation in lymphoid organs such as adjacent lymph nodes $\left(r_{s}=0.92, P<0.001\right)$ and spleen $\left(r_{s}=0.83\right.$, $P=0.005$ ), supporting the role of CXCR4-targeted imaging in visualizing both the regional and the systemic inflammatory response.

Knowledge about the presence of allograft involvement is crucial to initiate adequate therapy to prevent loss of graft function. In the context of immunosuppression, both clinical presentation and classic ultrasound signs may be less helpful to establish the diagnosis than in other clinical contexts. Furthermore, exclusion of infection in the native kidneys or in a remaining nonfunctional renal allograft may prevent unnecessary nephrectomy and surgery-related complications in these patients. When regarding the clinical impact of combined PET/MR, imaging facilitated clinical decisions (antibiotic treatment duration and regime, surgical options) in patients by localizing the site of infection. Because imaging showed the absence of infection of the native kidney or the nonfunctional first renal allograft in 12 of 13 patients, clinicians refrained from previously discussed nephrectomy, whereas in the other patient with infection of the polycystic kidney nephrectomy was suggested.

The inclusion of MRI data, providing high soft-tissue contrast images, was particularly helpful for interpretation of CXCR4 imaging. In particular, tracer accumulation in parts of the renal pelvis may be easily differentiated from cortical uptake using T2weighted MR sequences. Diffusion-weighted MRI has high sensitivity to detect areas of restricted diffusion in renal parenchyma indicating pyelonephritis (15). In particular, MRI facilitates the discrimination of foci with leukocyte infiltration from normal parenchyma because the range of ADC values in normal parenchyma is narrow and, in contrast to SUVs, there is no overlap for $\mathrm{ADC}_{\text {min }}$ and only little overlap for $\mathrm{ADC}_{\text {mean }}$ between foci and parenchyma (Fig. 1). Thus, clear visualization of increased cell density in areas of ADC reduction improved the interpretation of PET images and supported the reader confidence when interpreting $\mathrm{CXCR}^{+}$foci. Multiparametric MRI may provide an even more comprehensive assessment of allografts, targeting additional pathophysiologic aspects of allograft infection and dysfunction that cannot be evaluated by CXCR4-targeted PET, including regional perfusion, tubular damage, and graft function $(16,17)$. In summary, advantages of MRI include the potential to assess functional pathophysiologic aspects beyond CXCR4 expression determined by PET and to confirm increased cell density in areas of ${ }^{68} \mathrm{Ga}$-pentixafor uptake. MRI alone, which has high sensitivity for cortical lesions, can nevertheless not reliably differentiate between active infectious foci and other causes of ADC reduction, for example, cortical scarring, and PET/CT readily demonstrates active inflammation. Therefore, there is a complementary role of PET and MRI in the context of assessment of allografts.

Some limitations of the present study should be acknowledged. First, the number of included patients is relatively small in this first pilot study, limiting the statistical power to detect significant associations with laboratory values, which would possibly be observed in larger cohorts. However, the observed results are promising, consistently observed across different imaging methods and validated by clinical data. In addition, the cohort included in this study is relatively heterogeneous, for example, in terms of time since first transplantation and prior antibiotic therapy. Although these parameters reflect clinical reality, the high sensitivity for detecting graft infection and infectious foci outside the kidney needs to be confirmed in larger prospective studies. Second, allograft biopsy was performed in only 2 patients, because biopsies are usually contraindicated in UTI and performed only in rare cases of suspected concomitant rejection. Finally, the precise cell population contributing to the in vivo ${ }^{68} \mathrm{Ga}$-pentixafor signal cannot be identified. Given the fact that CXCR4 is expressed by a variety of immune cells $(10,21,22)$ and that allograft biopsy confirmed accumulation of different types of immune cells, uptake of ${ }^{68} \mathrm{Ga}$-pentixafor represents an inflammatory composite signal. CXCR4-targeted PET imaging can therefore be regarded as a sensitive tool for specific visualization of leukocytes. However, further studies including patients with allograft rejection are desirable to investigate whether the intensity or pattern of ${ }^{68} \mathrm{Ga}$-pentixafor accumulation may be used to differentiate between the presence of infection and other pathologies in which infiltrates of $\mathrm{CXCR}^{+}{ }^{+}$immune cells are involved, for example, rejection.

\section{CONCLUSION}

Combined CXCR4-targeted PET/MRI with ${ }^{68} \mathrm{Ga}$-pentixafor may enable the noninvasive detection of leukocytes in renal allografts. This novel methodology may refine the characterization of infectious and inflammatory kidney diseases and may serve as a platform for future clinical studies targeting kidney or kidney allograft infection.

\section{DISCLOSURE}

Hans-Jürgen Wester is a shareholder of Scintomics. No other potential conflict of interest relevant to this article was reported. 


\section{REFERENCES}

1. de Souza RM, Olsburgh J. Urinary tract infection in the renal transplant patient. Nat Clin Pract Nephrol. 2008;4:252-264.

2. Wu X, Dong Y, Liu Y, et al. The prevalence and predictive factors of urinary tract infection in patients undergoing renal transplantation: a meta-analysis. Am J Infect Control. 2016;44:1261-1268.

3. Chuang P, Parikh CR, Langone A. Urinary tract infections after renal transplantation: a retrospective review at two US transplant centers. Clin Transplant. 2005;19:230-235.

4. Gołębiewska J, Dębska-Ślizień A, Zadrożny D, et al. Acute graft pyelonephritis during the first year after renal transplantation. Transplant Proc. 2014;46:2743-2747.

5. Abbott KC, Swanson SJ, Richter ER, et al. Late urinary tract infection after renal transplantation in the United States. Am J Kidney Dis. 2004;44:353-362.

6. Pellé G, Vimont S, Levy PP, et al. Acute pyelonephritis represents a risk factor impairing long-term kidney graft function. Am J Transplant. 2007;7:899-907.

7. Dupont PJ, Psimenou E, Lord R, Buscombe JR, Hilson AJ, Sweny P. Late recurrent urinary tract infections may produce renal allograft scarring even in the absence of symptoms or vesicoureteric reflux. Transplantation. 2007;84:351-355.

8. Gourni E, Demmer O, Schottelius M, et al. PET of CXCR4 expression by a ${ }^{68} \mathrm{Ga}-$ labeled highly specific targeted contrast agent. J Nucl Med. 2011;52:1803-1810.

9. Herrmann K, Lapa C, Wester HJ, et al. Biodistribution and radiation dosimetry for the chemokine receptor CXCR4-targeting probe ${ }^{68} \mathrm{Ga}$-pentixafor. $\mathrm{J} \mathrm{Nucl}$ Med. 2015;56:410-416.

10. Mahalingam S, Karupiah G. Chemokines and chemokine receptors in infectious diseases. Immunol Cell Biol. 1999;77:469-475.

11. Wester HJ, Keller U, Schottelius M, et al. Disclosing the CXCR4 expression in lymphoproliferative diseases by targeted molecular imaging. Theranostics. 2015;5:618-630.

12. Philipp-Abbrederis K, Herrmann K, Knop S, et al. In vivo molecular imaging of chemokine receptor CXCR4 expression in patients with advanced multiple myeloma. EMBO Mol Med. 2015;7:477-487.

13. Thackeray JT, Derlin T, Haghikia A, et al. Molecular imaging of the chemokine receptor CXCR4 after acute myocardial infarction. JACC Cardiovasc Imaging. 2015;8:1417-1426.
14. Hyafil F, Pelisek J, Laitinen I, et al. Imaging the cytokine receptor CXCR4 in atherosclerotic plaques with the radiotracer ${ }^{68} \mathrm{Ga}$-pentixafor for positron emission tomography. J Nucl Med. 2017;58:499-506.

15. Faletti R, Cassinis MC, Gatt IM, et al. Acute pyelonephritis in transplanted kidneys: can diffusion-weighted magnetic resonance imaging be useful for diagnosis and follow-up? Abdom Radiol (NY). 2016;41:531-537.

16. Hueper K, Hensen B, Gutberlet M, et al. Kidney transplantation: multiparametric functional magnetic resonance imaging for assessment of renal allograft pathophysiology in mice. Invest Radiol. 2016;51:58-65.

17. Hueper K, Khalifa AA, Bräsen JH, et al. Diffusion-weighted imaging and diffusion tensor imaging detect delayed graft function and correlate with allograft fibrosis in patients early after kidney transplantation. J Magn Reson Imaging. 2016;44:112-121.

18. Levey AS, Stevens LA, Schmid CH, et al. A new equation to estimate glomerular filtration rate. Ann Intern Med. 2009;150:604-612.

19. Martin R, Jüttler S, Müller M, Wester HJ. Cationic eluate pretreatment for automated synthesis of $\left[{ }^{68} \mathrm{Ga}\right]$ CPCR4.2. Nucl Med Biol. 2014;41:84-89.

20. Demmer O, Dijkgraaf I, Schumacher U, et al. Design, synthesis, and functionalization of dimeric peptides targeting chemokine receptor CXCR4. J Med Chem. 2011;54:7648-7662.

21. Eash KJ, Means JM, White DW, et al. CXCR4 is a key regulator of neutrophil release from the bone marrow under basal and stress granulopoiesis conditions. Blood. 2009;113:4711-4719.

22. Gupta SK, Pillarisetti K, Lysko PG. Modulation of CXCR4 expression and SDF-1 alpha functional activity during differentiation of human monocytes and macrophages. J Leukoc Biol. 1999;66:135-143.

23. Besson FL, Chaumet-Riffaud P, Playe M, et al. Contribution of ${ }^{18}$ F-FDG PET in the diagnostic assessment of fever of unknown origin (FUO): a stratification-based meta-analysis. Eur J Nucl Med Mol Imaging. 2016;43:18871895.

24. McCammack KC, Hawkes NC, Silverman ED, et al. PET/CT appearance of acute pyelonephritis. Clin Nucl Med. 2013;38:e299-e301.

25. Derlin T, Weiberg D, von Klot C, et al. ${ }^{68}$ Ga-PSMA I\&T PET/CT for assessment of prostate cancer: evaluation of image quality after forced diuresis and delayed imaging. Eur Radiol. 2016;26:4345-4353. 